\title{
Discrete Fourier transform based pattern classifiers
}

\author{
S. HUI ${ }^{1 *}$ and S.H. ŻAK ${ }^{2}$ \\ ${ }^{1}$ Department of Mathematical Sciences, San Diego State University, San Diego, CA 92182, USA \\ ${ }^{2}$ School of Electrical and Computer Engineering, Purdue University, West Lafayette, IN 47907, USA
}

\begin{abstract}
A technique for pattern classification using the Fourier transform combined with the nearest neighbor classifier is proposed. The multidimensional fast Fourier transform (FFT) is applied to the patterns in the data base. Then the magnitudes of the Fourier coefficients are sorted in descending order and the first $P$ coefficients with largest magnitudes are selected, where $P$ is a design parameter. These coefficients are then used in further processing rather than the original patterns. When a noisy pattern is presented for classification, the pattern's $P$ Fourier coefficients with largest magnitude are extracted. The coefficients are arranged in a vector in the descending order of their magnitudes. The obtained vector is referred to as the signature vector of the corresponding pattern. Then the distance between the signature vector of the pattern to be classified and the signature vectors of the patterns in the data base are computed and the pattern to be classified is matched with a pattern in the data base whose signature vector is closest to the signature vector of the pattern being classified.
\end{abstract}

Key words: pattern classification, multidimensional discrete Fourier transform (DFT), Fourier coefficients.

\section{Introduction}

An essential element of a quality pattern classifier is a feature extraction algorithm that is capable of extracting features that are invariant to certain geometric transformations. In the paper, we focus on classifying images that are transformed from original prototype images by a group of planar transformations (see Subsec. 2.1). The Fourier transform possesses a number properties that make it suitable for invariant feature extraction for pattern recognition. Altmann and Reitböck [1] and Reitboeck and Altmann [2] proposed a size and positioninvariant description of an image function via the absolute value of the Mellin transform of its amplitude spectrum (the absolute value of the Fourier transform.) Gardenier, McCallum, and Bates [3] used the Fourier transform amplitudes in pattern recognition applications. More recently, Chen, Bui, and Krzyżak [4] employed the Radon transform and dualtree complex wavelets, in addition to Fourier transforms, in the invariant pattern recognition. The well-known Fourier descriptor method for the shape analysis and classification is fundamentally different from the proposed method; see Sec. 6 for a discussion.

The method we are proposing uses the amplitude spectra of the images. It is not immediately clear that the amplitude spectrum can uniquely determine the image. In other words, different functions may have the same amplitude spectrum. However, it is well-known that functions that arise in practice are uniquely determined by their amplitude spectra, see Barakat and Newsam [5], and Van Hove, Lim, and Oppenheim [6], and Taylor [7]. The study of the determination of a function, either continuous or discrete, from its amplitude spectrum has a long history, see for example, Akutowicz [8, 9], Barakat and Newsam [5], and Van Hove, Lim, and Oppenheim [6]. Many of the studies also discuss the possi- ble recovery of the function from its amplitude spectrum, see for example, Hayes, Lim, and Oppenheim [10], Hayes [11], Taylor [7], and Bates and McDonnell [12].

The feature of each image that we use in our method is the decreasing rearrangement of the amplitude spectrum of the image. There is no reason to believe that the decreasing rearrangement of the amplitude spectrum of an image can uniquely determine the image itself. However, it is our experience that the decreasing rearrangement of the amplitude spectrum does determine the image in all cases we have studied.

We propose an algorithm where $P$ Fourier coefficients with largest absolute values are extracted. The magnitudes of coefficients are arranged in a vector in descending order. We refer to the obtained vector as the signature vector of the corresponding pattern. The distance between the signature vector of the pattern to be classified and the signature vectors of the patterns in the data base are computed and the pattern to be classified is matched with a pattern in the data base whose signature vector is closest to the signature vector of the pattern being classified.

The paper is organized as follows. In Sec. 2, we present relevant background results related to the Fourier transform and its properties that we use to extract pattern features for the purpose of pattern classification. In Subsec. 3.2, we discuss the use of discrete Fourier coefficients as signature vectors in noisy environments. In Sec. 4, we propose a Fourier transform based algorithm for pattern classification. In Sec. 5, we present results of numerical experiments demonstrating the effectiveness of the proposed pattern classifier. A brief discussion of the difference between the proposed technique and the Fourier descriptor method is given in Sec. 6. Conclusions are found in Sec. 7.

*e-mail: shui@mail.sdsu.edu 


\section{Mathematical preliminaries}

In this section, we collect the mathematical results that we use in our discussion. First, recall that a standard rectangular RGB image is represented by a 3D matrix $x$ of size $M_{1} \times M_{2} \times 3$. Each of the three $M_{1} \times M_{2}$ submatrices contain the intensity values for red, green, and blue, respectively. We allow the following operations on the images: rotation through $90^{\circ}, 180^{\circ}, 270^{\circ}$, and reflections through the horizontal, median, the vertical median, and the two main diagonals of the image. Let $\Gamma$ denote the set of these operations. By combining the operations in $\boldsymbol{\Gamma}$, a total of 28 different images can be generated from a single image. Note that the operations in $\Gamma$ only affect the first two dimensions of an image. For $\gamma \in \boldsymbol{\Gamma}$, we use $\gamma(x)$ to denote the image obtained from $x$ using the operation $\gamma$.

Let $\ell$ and $m_{1}, \ldots, M_{\ell}$ be positive integers. For our applications, $\ell=3$ but we present the general case for notational convenience. Let $\boldsymbol{L} \in \mathbb{Z}^{\ell}$ be the rectangular lattice

$$
\boldsymbol{L}=\left[0, \ldots, M_{1}-1\right] \times \cdots \times\left[0, \ldots, M_{\ell}-1\right]
$$

and let $M=\left(M_{1}, \ldots, M_{\ell}\right)$ be the vector containing the dimensions of the lattice $\boldsymbol{L}$. Then the image $x$ can be viewed as a complex-valued function on $\boldsymbol{L}$, that is, $x: \boldsymbol{L} \rightarrow \mathbb{C}$.

2.1. The Discrete Fourier Transform (DFT). We first recall the following standard notation: Let $\boldsymbol{m}=\left(m_{1}, \ldots, m_{\ell}\right)$ and $\boldsymbol{M}=\left(M_{1}, \ldots, M_{\ell}\right)$. We denote the coordinate-wise division of $\boldsymbol{m}$ by $M$ by

$$
\frac{\boldsymbol{m}}{\boldsymbol{M}}=\left(\frac{m_{1}}{M_{1}}, \ldots, \frac{m_{\ell}}{M_{\ell}}\right) .
$$

The discrete Fourier transform (DFT) of $x$ is defined by

$$
\widehat{x}(\boldsymbol{n})=\sum_{\boldsymbol{m} \in \boldsymbol{L}} x(\boldsymbol{m}) e^{-2 \pi j \boldsymbol{n} \cdot \frac{\boldsymbol{m}}{\boldsymbol{M}}}
$$

for each $\boldsymbol{n} \in \boldsymbol{L}$. The basic properties of the Fourier transform can be found in [13-15].

We make use of the following properties of the DFT. The following theorem is well known:

Theorem 1. Let $x: L \rightarrow \mathbb{C}$. Then

$$
\|\widehat{x}\|^{2}=|M|\|x\|^{2},
$$

where $|\boldsymbol{M}|=M_{1} \cdots M_{\ell}$.

We also need the following result on the values of $\widehat{x}$. Its proof is elementary but it does not appear in the standard Fourier analysis books and so for the convenience of the reader, we have included its proof. For $x: \boldsymbol{L} \rightarrow \mathbb{C}$, let

$$
A(x)=\{|\widehat{x}(\boldsymbol{n})|: \boldsymbol{n} \in \boldsymbol{L}\}
$$

denote the set of values of the amplitude spectrum of $x$.

Theorem 2. For each $x: \boldsymbol{L} \rightarrow \mathbb{C}$ and each $\gamma \in \boldsymbol{\Gamma}$,

$$
A(x)=A(\gamma(x)) \text {. }
$$

Proof. Suppose $x$ has dimension $M_{1} \times M_{2} \times \cdots \times M_{\ell}$. Let $\tau(x)$ denote the vector obtained from $x$ by transposing its first two coordinates. Note that for an image $x, \tau(x)$ is the same as reflecting $x$ through its main diagonal. Then $\tau(x)$ has dimension $M_{2} \times M_{1} \times \cdots \times M_{\ell}$ and is a function on the transposed lattice

$$
\begin{gathered}
\boldsymbol{L}^{\prime}=\left[0, \ldots, M_{2}-1\right] \times\left[0, \ldots, M_{1}-1\right] \\
\times \cdots \times\left[0, \ldots, M_{\ell}-1\right] \\
=\left[0, \ldots, M_{2}-1\right] \times\left[0, \ldots, M_{1}-1\right] \times \boldsymbol{L}_{3},
\end{gathered}
$$

where $\boldsymbol{L}_{3}=\left[0, \ldots, M_{3}-1\right] \times \cdots \times\left[0, \ldots, M_{\ell}-1\right]$. We have for $\boldsymbol{n} \in \boldsymbol{L}^{\prime}$ that

$$
\begin{gathered}
\widehat{\tau(x)}(\boldsymbol{n})=\sum_{\boldsymbol{m}^{\prime} \in \boldsymbol{L}^{\prime}} \tau(x)\left(\boldsymbol{m}^{\prime}\right) e^{-2 \pi j \boldsymbol{n} \cdot \frac{\boldsymbol{m}}{\boldsymbol{M}}} \\
=\sum_{\boldsymbol{m}^{\prime \prime} \in \boldsymbol{L}_{3}} \sum_{m_{1}^{\prime}=0}^{M_{2}-1} \sum_{m_{2}^{\prime}=0}^{M_{1}-1} \tau(x)\left(m_{1}^{\prime}, m_{2}^{\prime}, \boldsymbol{m}^{\prime \prime}\right) e^{-2 \pi j\left(\frac{m_{1}^{\prime} n_{1}}{M_{1}}+\frac{m_{2}^{\prime} n_{2}}{M_{2}}+\boldsymbol{m}^{\prime \prime} \cdot \frac{\boldsymbol{n}^{\prime \prime}}{M}\right)} \\
=\sum_{\boldsymbol{m}^{\prime \prime} \in \boldsymbol{L}_{3}} \sum_{m_{1}^{\prime}=0}^{M_{2}-1} \sum_{m_{2}^{\prime}=0}^{M_{1}-1} x\left(m_{2}^{\prime}, m_{1}^{\prime}, \boldsymbol{m}^{\prime \prime}\right) e^{-2 \pi j\left(\frac{m_{2}^{\prime} n_{2}}{M_{2}}+\frac{m_{1}^{\prime} n_{1}}{M_{1}}+\boldsymbol{m}^{\prime \prime} \cdot \frac{\boldsymbol{n}^{\prime \prime}}{M}\right)} \\
=\widehat{x}\left(\boldsymbol{n}^{\prime}\right),
\end{gathered}
$$

where $\boldsymbol{n}^{\prime}$ denotes the vector obtained from $\boldsymbol{n}$ by transposing its first two coordinates. We conclude that the DFT commutes with transposition. It follows that

$$
A(x)=A(\tau(x)) \text {. }
$$

Let $\phi$ denote the reflection of the first two coordinates through the horizontal median. Then $\phi(x)$ has the same dimension as $x$ and for $0 \leq m_{1} \leq M_{1}-1$ and $m=\left(m_{1}, \boldsymbol{m}_{2}\right) \in \boldsymbol{L}$,

$$
\phi(x)\left(m_{1}, \boldsymbol{m}_{2}\right)=x\left(M_{1}-m_{1}-1, \boldsymbol{m}_{2}\right) .
$$

Factor the lattice $\boldsymbol{L}$ into $\boldsymbol{L}=\left[0, \ldots, M_{1}-1\right] \times \boldsymbol{L}_{2}$ and for $\boldsymbol{n} \in \boldsymbol{L}$, let $\boldsymbol{n}=\left(n_{1}, \boldsymbol{n}_{2}\right)$. Then

$$
\begin{gathered}
\widehat{\phi(x)}(\boldsymbol{n})=\sum_{\boldsymbol{m} \in \boldsymbol{L}} \phi(x)(\boldsymbol{m}) e^{-2 \pi j} \boldsymbol{n \cdot \frac { \boldsymbol { m } } { \boldsymbol { M } }} \\
=\sum_{\boldsymbol{m}_{2} \in \boldsymbol{L}_{2}} \sum_{m_{1}=0}^{M_{1}-1} \phi(x)\left(m_{1}, \boldsymbol{m}_{2}\right) e^{-2 \pi j\left(\frac{m_{1} n_{1}}{M_{1}}+\boldsymbol{n}_{2} \cdot \frac{\boldsymbol{m}_{2}}{\boldsymbol{M}_{2}}\right)} \\
=\sum_{\boldsymbol{m}_{2} \in \boldsymbol{L}_{2}} \sum_{m_{1}=0}^{M_{1}-1} x\left(M_{1}-m_{1}-1, \boldsymbol{m}_{2}\right) e^{-2 \pi j\left(\frac{m_{1} n_{1}}{M_{1}}+\boldsymbol{n}_{2} \cdot \frac{\boldsymbol{m}_{2}}{\boldsymbol{M}_{2}}\right)} \\
=\sum_{\boldsymbol{m}_{2} \in \boldsymbol{L}_{2}} \sum_{m=0}^{M_{1}-1} x\left(m, \boldsymbol{m}_{2}\right) e^{-2 \pi j}\left(\frac{\left(M_{1}-m-1\right) n_{1}}{M_{1}}+\boldsymbol{n}_{2} \cdot \frac{\boldsymbol{m}_{2}}{\boldsymbol{M}_{2}}\right) \\
=e^{-2 \pi j} \frac{\left(M_{1}-1\right) n_{1}}{M_{1}} \sum_{\boldsymbol{m}_{2} \in \boldsymbol{L}_{2}} \sum_{m=0}^{M_{1}-1} x\left(m, \boldsymbol{m}_{2}\right) e^{-2 \pi j\left(-\frac{m n_{1}}{M_{1}}+\boldsymbol{n}_{2} \cdot \frac{\boldsymbol{m}_{2}}{\boldsymbol{M}_{2}}\right)} \\
\sum_{\boldsymbol{m}_{2} \in \boldsymbol{L}_{2}} \sum_{m=0}^{M_{1}-1} x\left(m, \boldsymbol{m}_{2}\right) e^{-2 \pi j}\left(\frac{m\left(M_{1}-n_{1}\right)}{M_{1}}+\boldsymbol{n}_{2} \cdot \frac{\boldsymbol{m}_{2}}{\boldsymbol{M}_{2}}\right) \\
=e^{2 \pi j \frac{n_{1}}{M_{1}} \widehat{x}\left(M_{1}-n_{1}, \boldsymbol{n}_{2}\right) .}
\end{gathered}
$$

The next to last equality is obtained using the fact that $e^{2 \pi j k}=$ 1 for all integers $k$. It follows that for $\boldsymbol{n}=\left(n_{1}, \boldsymbol{n}_{2}\right) \in \boldsymbol{L}$,

$$
|\widehat{\phi(x)}(\boldsymbol{n})|= \begin{cases}\left|\widehat{x}\left(0, \boldsymbol{n}_{2}\right)\right| & \text { if } n_{1}=0 \\ \left|\widehat{x}\left(M_{1}-n_{1}, \boldsymbol{n}_{2}\right)\right| & \text { if } 1 \leq n_{1} \leq M_{1}-1 .\end{cases}
$$

Thus the magnitude of the DFT commutes with reflection through the horizontal median and so $A(x)=A(\phi(x))$. The 
other operations in $\Gamma$ can all be expressed as combinations of $\tau$ and $\phi$ :

1. $\rho=$ Counter-clockwise rotation by $90^{\circ}=\phi \circ \tau$

2. $\nu=$ Reflection through vertical median $=\rho^{3} \circ \phi \circ \rho$

3. Reflection through opposite diagonal $=\nu \circ \tau \circ \nu$

It follows that $A(x)$ is invariant under operations in $\boldsymbol{\Gamma}$.

Theorem 2 shows that the magnitude of the DFT is invariant under the operations in $\Gamma$. This property makes the magnitude of the DFT an attractive tool in the design of recognition algorithms that are robust against the operations in $\Gamma$. However, we cannot use the magnitude of the DFT directly as it is not invariant under the operations in $\Gamma$; only the unordered values are. We show in Subsec. 3.2 that there are drawbacks to using the entire DFT in noisy environments.

2.2. Rearrangement of vectors. In our algorithm, we arrange the values of $A(x)$ in decreasing order of magnitude. In this section, we state and prove two simple results on rearranged vectors. The results are classical and we include their proofs since they do not seem to be widely known outside of classical analysis.

Lemma 3. Suppose $\boldsymbol{a}$ and $\boldsymbol{b}$ are real vectors of the length $N$. Then the maximum of the dot products of all possible rearrangements of $\boldsymbol{a}$ and $\boldsymbol{b}$ is achieved when the values in each vector are arranged in decreasing order.

Proof. Let $\boldsymbol{a}$ and $\boldsymbol{b}$ be real vectors of length $N$. If $a_{1}=\cdots=$ $a_{N}$, then the claim is certainly true because all rearrangements of $\boldsymbol{b}$ give the same dot product. We can assume without loss of generality that $a_{1} \geq a_{2} \geq \cdots \geq a_{N}$. and that not all $a_{k}$ 's are the same. Let $s$ be the maximum of the dot products. Suppose

$$
s=a_{1} b_{1}^{\prime}+\cdots+a_{N} b_{N}^{\prime}
$$

for the rearrangement $\left(b_{1}^{\prime}, \ldots, b_{N}^{\prime}\right)$ of $\left(b_{1}, \ldots, b_{N}\right)$. Suppose $j<k$ and $a_{j}>a_{k}$. Then we must have $b_{j}^{\prime} \geq b_{k}^{\prime}$ because otherwise we have

$$
a_{j} b_{k}^{\prime}+a_{k} b_{j}^{\prime}-\left(a_{j} b_{j}^{\prime}+a_{k} b_{k}^{\prime}\right)=\left(a_{j}-a_{k}\right)\left(b_{k}^{\prime}-b_{j}^{\prime}\right)>0
$$

and we can obtain a larger dot product by switching $b_{j}^{\prime}$ and $b_{k}^{\prime}$. The claim now follows by rearranging the $b_{k}$ 's in a range where the $a_{k}$ 's are constant in decreasing order.

The following is now immediate.

Theorem 4. Let $\boldsymbol{a}$ and $\boldsymbol{b}$ be real vectors of length $N$ and let $\boldsymbol{a}^{\sharp}$ and $\boldsymbol{b}^{\sharp}$ be the rearrangements of $\boldsymbol{a}$ and $\boldsymbol{b}$ in decreasing order. Then

$$
\left\|a^{\sharp}-b^{\sharp}\right\| \leq\|\boldsymbol{a}-\boldsymbol{b}\| .
$$

Proof. Since $\left\|\boldsymbol{a}^{\sharp}\right\|^{2}=\|\boldsymbol{a}\|^{2},\left\|\boldsymbol{b}^{\sharp}\right\|^{2}=\|\boldsymbol{b}\|^{2}$, and $\boldsymbol{a}^{\sharp} \cdot \boldsymbol{b}^{\sharp} \geq \boldsymbol{a} \cdot \boldsymbol{b}$, we have

$$
\begin{gathered}
\left\|\boldsymbol{a}^{\sharp}-\boldsymbol{b}^{\sharp}\right\|^{2}=\left\|\boldsymbol{a}^{\sharp}\right\|^{2}-\boldsymbol{a}^{\sharp} \cdot \boldsymbol{b}^{\sharp}+\left\|\boldsymbol{b}^{\sharp}\right\|^{2} \\
\leq\|\boldsymbol{a}\|^{2}-\boldsymbol{a} \cdot \boldsymbol{b}+\|\boldsymbol{b}\|^{2}=\|\boldsymbol{a}-\boldsymbol{b}\| .
\end{gathered}
$$

\section{The pattern recognition algorithm}

In this section, we give the motivation for the algorithm based on the mathematical results given in Sec. 2 and the impact of noise (see Subsec. 3.2).

3.1. Motivation. Let $x_{1}, \ldots, x_{N}: \boldsymbol{L} \rightarrow \mathbb{R}$ be the pixel values of $N$ distinct $\ell$-dimensional prototype images. The original image for a received noiseless $y$ that has not been rotated or reflected can be recovered exactly by solving

$$
x_{\text {est }}=\arg \min _{k=1, \ldots, N}\left\|y-x_{k}\right\|_{2} \text {. }
$$

However, if the image has been rotated or reflected, then this method does not work. One approach would be to compare the received image to all possible images obtainable from the original set of prototype images. While this can be done, it increases the computational load almost 30 fold, which makes this approach less desirable.

Since the DFT is not invariant under rotation and reflection, using the DFT directly suffers the same problem as using the original image values. However, as we proved in Theorem 2 , the set of values of the amplitude spectrum

$$
A(x)=\{|\widehat{x}(\boldsymbol{m})|: \boldsymbol{m} \in \boldsymbol{L}\}
$$

is invariant under operations in $\boldsymbol{\Gamma}$. So we need to find a metric on sets that measures the difference between $A(x)$ and $A(y)$, and then use that to measure the difference between the images $x$ and $y$. The method we chose is based on Theorem 4 . Let $A^{\sharp}(x)$ denote the vector obtained from $A(x)$ by decreasing rearrangement. From Theorem 4 and Theorem 1, we have

$$
\left\|A^{\sharp}(x)-A^{\sharp}(y)\right\| \leq\|\widehat{x}-\widehat{y}\|=\sqrt{|\boldsymbol{M}|}\|x-y\| .
$$

Let

$$
d(x, y)=\left\|A^{\sharp}(x)-A^{\sharp}(y)\right\| .
$$

Then it is easy to see that $d$ is a semi-metric on the set of prototype images but it is not a metric on the set of all possible images. It is not a metric because it is possible to have $A^{\sharp}(x)=A^{\sharp}(y)$ even if $x \neq y$, but this rarely happens in practice for a finite set of images.

Remark 1. We can formalize the way images can be distinguished using the following equivalence relation. We say that the images $x$ and $y$ are equivalent, denoted by $x \sim y$, if $A(x)=A(y)$. It can easily be verified that $\sim$ is an equivalence relation on the space $\mathcal{I}$ of images with the same number of pixels. For each image $x$, let $[x]$ denote the equivalence class containing $x$. On the space $\mathcal{I} / \sim$ of equivalence classes, let

$$
D([x],[y])=d(x, y),
$$

where $d$ is the semi-metric defined in Eq. (4). Then $D$ is a metric on $\mathcal{I} / \sim$.

We assume that our prototype images have distinct equivalence classes. Then for $x \neq y, d(x, y)=D([x],[y])>0$ and so $d$ is a metric on the set of prototype images.

For a received image $y, d(x, y)>0$ if $x \neq y$ and the only solution of

$$
x_{\text {est }}=\arg \min _{k=1, \ldots, N} d\left(x_{k}, y\right) .
$$


is $x=y$, the original message. We have thus found a method for recovering noiseless images that have been transformed by the operations in $\Gamma$.

We next discuss the case when there is noise.

3.2. Noise considerations. Let $x_{1}, \ldots, x_{N}: \boldsymbol{L} \rightarrow \mathbb{R}$ be the pixel values of $N \ell$-dimensional prototype images. Note that standard color images are three-dimensional. The noisy version of the $k$-th image has the form

$$
y_{k}=x_{k}+\omega_{k}
$$

where $\omega_{k}$ a random variable with zero mean and finite variance $\sigma^{2}$ per pixel. Furthermore, we assume that $\left\{\omega_{k}(\boldsymbol{m})\right.$ : $k=1, \ldots, N, \boldsymbol{m} \in \boldsymbol{L}\}$ are independent and identically distributed (iid) random variables. Note that other than the properties stated above, the iid random variables can have any distribution. We will use zero mean Gaussian noise in the simulations but the analysis applies to all distributions that satisfy the above requirements.

The maximum likelihood estimator for a received noisy image $y$ is

$$
x_{e s t}=\arg \min _{k=1, \ldots, N}\left\|y-x_{k}\right\|_{2} .
$$

However, as we had seen before, the above estimator is not robust against rotation or translation and we chose instead to use

$$
x_{e s t}=\arg \min _{k=1, \ldots, N} d\left(x_{k}, y\right)
$$

where $d(x, y)=\left\|A^{\sharp}(x)-A^{\sharp}(y)\right\|$. If the noise is zero, then the actual image is a solution to the above optimization problem, and would be the unique solution when $d$ is a metric. However, as we next demonstrate, there is drawback to this approach in a noisy environment.

Let $x$ be a prototype image and $y=x+\omega$ a noisy version of the image. The $\boldsymbol{n}$-th Fourier coefficient of $y$ satisfies

$$
\begin{aligned}
&|\widehat{y}(\boldsymbol{n})|^{2}=\left|\sum_{\boldsymbol{m} \in \boldsymbol{L}}(x(\boldsymbol{m})+\omega(\boldsymbol{m})) e^{-2 \pi j} \boldsymbol{n} \cdot \frac{\boldsymbol{m}}{\boldsymbol{M}}\right|^{2} \\
&= \sum_{\boldsymbol{k}, \boldsymbol{m} \in \boldsymbol{L}} x(\boldsymbol{m}) \overline{x(\boldsymbol{k})} e^{-2 \pi j(\boldsymbol{m}-\boldsymbol{k}) \cdot \frac{\boldsymbol{n}}{M}} \\
&+2 \operatorname{Re}\left(\sum_{\boldsymbol{k}, \boldsymbol{m} \in \boldsymbol{L}} x(\boldsymbol{m}) \overline{\omega(\boldsymbol{k})} e^{-2 \pi j(\boldsymbol{m}-\boldsymbol{k}) \cdot \frac{\boldsymbol{n}}{M}}\right) \\
&+\sum_{\boldsymbol{k}, \boldsymbol{m} \in \boldsymbol{L}} \omega(\boldsymbol{m}) \overline{\omega(\boldsymbol{k})} e^{-2 \pi j(\boldsymbol{m}-\boldsymbol{k}) \cdot \frac{\boldsymbol{n}}{M}} \\
&=|\widehat{x}(\boldsymbol{n})|^{2}+ \\
&+\sum_{\boldsymbol{k}, \boldsymbol{m} \in \boldsymbol{L}}\left(\sum_{\boldsymbol{k}, \boldsymbol{m} \in \boldsymbol{L}} x(\boldsymbol{m}) \overline{\omega(\boldsymbol{m})} \overline{\omega(\boldsymbol{k})} e^{-2 \pi j(\boldsymbol{m}-\boldsymbol{k}) \cdot \frac{\boldsymbol{n}}{M}}\right.
\end{aligned}
$$

The expected value of $|\widehat{y}(\boldsymbol{n})|^{2}$ is

$$
\begin{gathered}
\boldsymbol{E}\left[|\widehat{y}(\boldsymbol{n})|^{2}\right]=|\widehat{x}(\boldsymbol{n})|^{2} \\
+\boldsymbol{E}\left[2 \operatorname{Re}\left(\sum_{\boldsymbol{k}, \boldsymbol{m} \in \boldsymbol{L}} x(\boldsymbol{m}) \overline{\omega(\boldsymbol{k})} e^{-2 \pi j(\boldsymbol{m}-\boldsymbol{k}) \cdot \frac{\boldsymbol{n}}{M}}\right)\right. \\
\left.+\sum_{\boldsymbol{k}, \boldsymbol{m} \in \boldsymbol{L}} \omega(\boldsymbol{m}) \overline{\omega(\boldsymbol{k})} e^{-2 \pi j(\boldsymbol{m}-\boldsymbol{k}) \cdot \frac{\boldsymbol{n}}{\boldsymbol{M}}}\right] .
\end{gathered}
$$

Since $\omega(\boldsymbol{m})$ and $\omega(\boldsymbol{k})$ are independent with mean zero and variance $\sigma^{2}$, we have

$$
\begin{gathered}
\boldsymbol{E}\left[|\widehat{y}(\boldsymbol{n})|^{2}\right]=|\widehat{x}(\boldsymbol{n})|^{2} \\
+\sum_{\boldsymbol{k}=\boldsymbol{m} \in \boldsymbol{L}} \boldsymbol{E}[\omega(\boldsymbol{m}) \overline{\omega(\boldsymbol{k})}] e^{-2 \pi j(\boldsymbol{m}-\boldsymbol{k}) \cdot \frac{\boldsymbol{n}}{\boldsymbol{M}}} \\
=|\widehat{x}(\boldsymbol{n})|^{2}+|\boldsymbol{M}| \sigma^{2}
\end{gathered}
$$

where $|\boldsymbol{M}|=M_{1} \cdots M_{\ell}$ is the number of pixels in the image. In light of Eq. (10), we see that a particular Fourier coefficient $\widehat{x}(\boldsymbol{n})$ is useful as a feature only if $|\widehat{x}(\boldsymbol{n})|^{2}$ is significantly greater than $M \sigma^{2}$. For most practical images with a moderate number of pixels, the number of Fourier coefficients whose squared-amplitudes are greater than two or three times $M \sigma^{2}$ is quite small for any nontrivial noise variance $\sigma^{2}$. Note this observation comports with the Riemann-Lebesgue lemma, see for example [13, p. 39]. We illustrate this crucial point with a numerical example (Fig. 1).

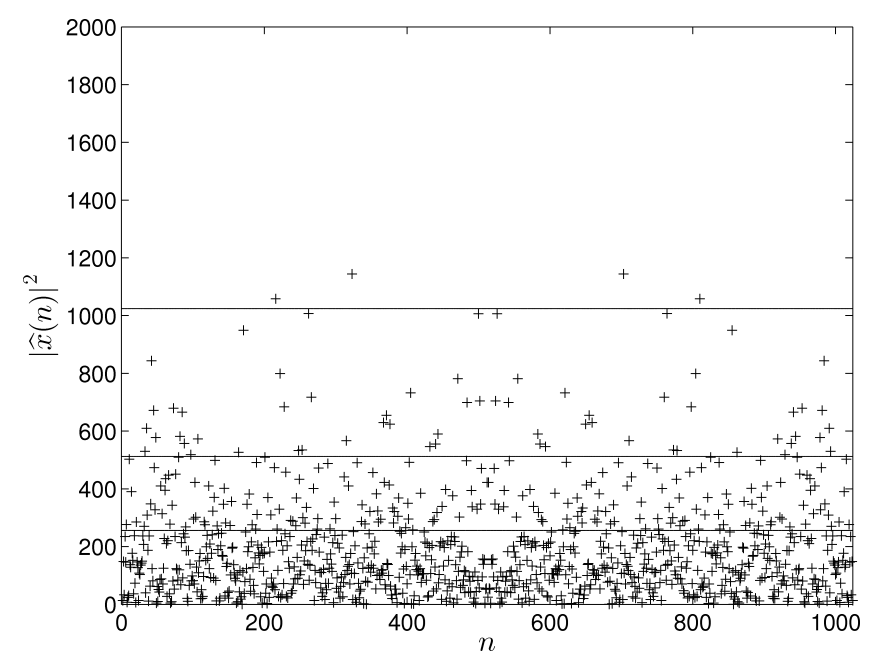

Fig. 1. A plot of the magnitude squared of the Fourier coefficients in Example 1.

Example 1. We generate a random zero-one vector $x$ of length 1024 where each entry has probability 0.25 of being one. We compute the Fourier coefficients of $x$ and plot $|\widehat{x}(n)|^{2}$ as a function of $n$. The three horizontal lines are at height $0.25 \times 1024,0.5 \times 1024$, and 1024 , respectively. The plot was generated by the following simple Matlab script:

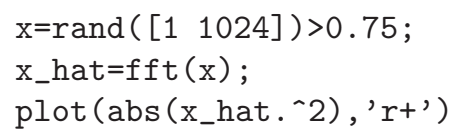


$\operatorname{axis}\left(\left[\begin{array}{llll}0 & 1024 & 0 & 2 * 10^{\wedge} 3\end{array}\right]\right)$

hold on

plot $(1: 1024,1024 * 0.25)$

plot $(1: 1024,1024 * 0.5)$

plot $(1: 1024,1024 * 1)$

hold off

Note that the plot excludes the high DC component at frequency 0 . This is accomplished in the above script using the command axis ([ [ $\left.\left.\begin{array}{llll}0 & 1024 & 0 & 2 * 10^{\wedge} 3\end{array}\right]\right)$.

The significance of Eq. (10) is that it implies that most of Fourier coefficients are not useful as features of an image in a noisy environment. In fact, the difference $d\left(x_{k}, y\right)$ in Eq. (9) may be overwhelmed by noise. It therefore makes sense to only consider the Fourier coefficients with the largest magnitudes. Note that using a scaled version of the DFT, for example,

$$
\widehat{x}(\boldsymbol{n})=\frac{1}{M} \sum_{\boldsymbol{m} \in \boldsymbol{L}} x(\boldsymbol{m}) e^{-\frac{2 \pi j \boldsymbol{m} \cdot \boldsymbol{n}}{M}},
$$

does not alleviate the problem because all coefficients are scaled the same and so the signal-to-noise ratio does not change.

In light of the above discussion, we use only the largest values in $A^{\sharp}(x)$. Let $A_{P}^{\sharp}(x)$ be the vector containing the first $P$ coordinates of $A^{\sharp}(x)$ and let $d_{P}(x, y)=\left\|A_{p}^{\sharp}(x)-A_{P}^{\sharp}(y)\right\|$. In all practical situations, $d_{P}$ is a metric on the set of prototype images and we solve

$$
x_{\text {est }}=\arg \min _{k=1, \ldots, N} d_{P}\left(x_{k}, y\right)
$$

to find the most likely original image given the received image $y$.

3.3. Error analysis. In this section, we analyze the effect of noise on the proposed algorithm. Our main tool is Eq. (3). As in Subsec. 3.2, we assume that the noisy version of the $k$-th image has the form

$$
y_{k}=x_{k}+\omega_{k},
$$

where $\omega_{k}$ a random variable with zero mean and finite variance $\sigma^{2}$ per pixel. We have from Eq. (3) that

$$
\begin{gathered}
d^{2}(x, x+\omega)=\left\|A^{\sharp}(x)-A^{\sharp}(x+\omega)\right\|^{2} \leq\|\widehat{\omega}\|^{2} \\
=|M|\|\omega\|^{2}
\end{gathered}
$$

and it follows that the expected value of the squared error for the whole image satisfies

$$
\begin{gathered}
E\left[\left\|A^{\sharp}(x)-A^{\sharp}(x+\omega)\right\|^{2}\right] \leq|\boldsymbol{M}| E\left[\|\omega\|^{2}\right] \\
=|\boldsymbol{M}| \sum_{\boldsymbol{m} \in \boldsymbol{L}} E\left[|\omega(\boldsymbol{m})|^{2}\right] \\
=|\boldsymbol{M}| \sum_{\boldsymbol{m} \in \boldsymbol{L}} \sigma^{2}=|\boldsymbol{M}|^{2} \sigma^{2} .
\end{gathered}
$$

In the above computation, we used the fact that $\sigma^{2}=$ $E\left[|\omega(\boldsymbol{m})|^{2}\right]$ is the noise variance for each pixel. Since there are $|M|$ pixels in each image, the expected squared error per pixel is

$$
\frac{1}{|\boldsymbol{M}|} E\left[\left\|A^{\sharp}(x)-A^{\sharp}(x+\omega)\right\|^{2}\right]=|\boldsymbol{M}| \sigma^{2} .
$$

When compared with the un-rearranged per pixel error given in Eq. (10), we can see that rearrangement does not increase the per pixel mean-square error of the DFT.

We have for a fixed image $x$ and a noise sample $\omega$ that

$$
\begin{gathered}
\left\|A^{\sharp}(x)-A^{\sharp}(x+\omega)\right\|^{2} \leq|\boldsymbol{M}|\|\omega\|^{2} \\
=|\boldsymbol{M}|^{2}\left(\frac{1}{|\boldsymbol{M}|} \sum_{\boldsymbol{m} \in \boldsymbol{L}}|\omega(\boldsymbol{m})|^{2}\right) .
\end{gathered}
$$

\section{Pattern recognition algorithm}

The pattern recognition method we propose uses $P$ leading elements of the decreasing rearrangements of the magnitude of the Fourier coefficients of the images as signatures. The number $P$ is a design parameter chosen on the basis of nature of the prototype images and the expected range of noise variance. The complete algorithm can be summarized as follows:

1. Prototype Image Signature Extraction

(a) Fix a design parameter $P$.

(b) For each image given by a function $x$ defined on a lattice $\boldsymbol{L}$, compute its discrete Fourier transform $\widehat{x}$.

(c) Evaluate and sort $\{|\widehat{x}(\boldsymbol{n})|: \boldsymbol{n} \in \boldsymbol{L}\}$ in descending order.

(d) Store the $P$ highest values in a vector as the signature for the image.

2. Noisy Image Pattern Classification

(a) For a noisy image $y$ to be classified, repeat steps $1 \mathrm{~b}$, $1 \mathrm{c}$, and $1 \mathrm{~d}$ described above.

(b) From the stored prototype signature vectors, find the one with smallest distance from the signature vector of $y$.

Note that one can use any metric on $\mathbb{R}^{P}$ as the distance function in step 2 b. In our simulations, we use the $\ell^{1}$ norm, which gives almost the same performance as the $\ell^{2}$ norm.

\section{Simulations}

For our simulations, we used 14 color images of stamps from [16] that contain images of butterflies. Each image contains $300 \times 200$ color pixels, which we treat as a function on a $300 \times 200 \times 3$ integer lattice. The original pixel values were integers from 0 to 255 , which we converted into floating point real numbers with values between 0 and 1 . We computed the three dimensional discrete Fourier transform of each image and then sorted the magnitudes of the Fourier coefficients. We kept the top 30 magnitudes from each image as its signature. 
For the simulations, one of the 14 prototype images was randomly and uniformly selected. Zero mean Gaussian noise was added independently to each pixel to the selected image; see Fig. 2 for an illustration of a typical prototype image and its noisy version for selected noise levels. We use the standard definition of signal-to-noise ratio (SNR) for an image $x$ :

$$
\mathrm{SNR}=\sqrt{\frac{1}{|\boldsymbol{M}| \sigma^{2}} \sum_{\boldsymbol{n} \in \boldsymbol{L}}[x(\boldsymbol{n})-\bar{x}]^{2}}
$$

where $\sigma^{2}$ is the variance of the noise per pixel and $\bar{x}=$ $\sum_{\boldsymbol{n} \in \boldsymbol{L}} x(\boldsymbol{n}) / M$ is the average pixel value of the image $x$; see for example Chen, Bui, and Krzyzak [4]. Note that the above definition of SNR is independent of image size and can be interpreted as the image SNR and as the average per pixel SNR.

a) Sample image

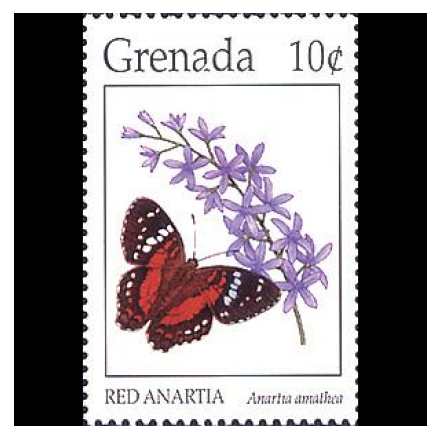

c) $\mathrm{SNR}=-3.5 \mathrm{~dB}$

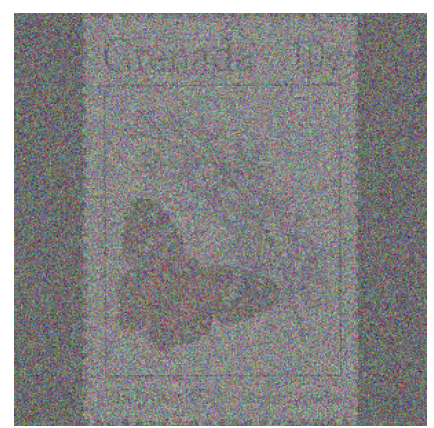

d) $\mathrm{SNR}=-6.5 \mathrm{~dB}$

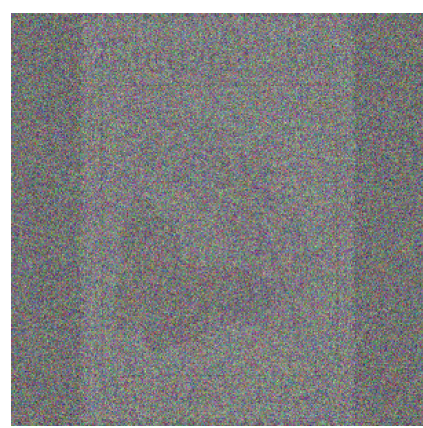

Fig. 2. Original image and its noisy versions used in the numerical experiment

The noisy image was rotated randomly and uniformly by $0^{\circ}, 90^{\circ}, 180^{\circ}$, or $270^{\circ}$; see Fig. 3 for an illustration of the rotations of a typical image. The signature vector of the noisy rotated image was extracted and the prototype image with the closest signature vector in $\ell^{1}$ norm was selected as the best estimate. The number of errors was recorded. Note that there are a total of 56 possible images without noise.

We repeated the same experiment using compressed versions of the same images. Each of three color components of an image was compressed by a two dimensional Daubechies wavelet to have size $75 \times 50$. The three compressed color components combine to form a compressed color image of

size $75 \times 50 \times 3$; see Fig. 4 for a typical compressed image and selected noisy versions.

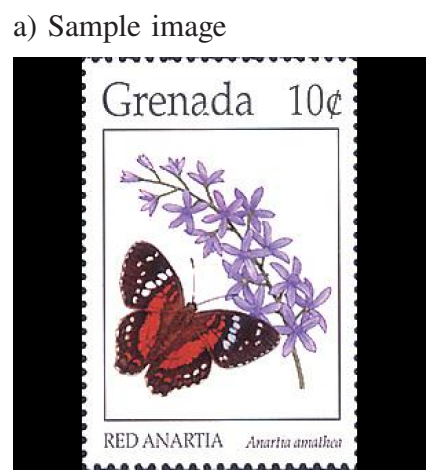

b) $90^{\circ}$

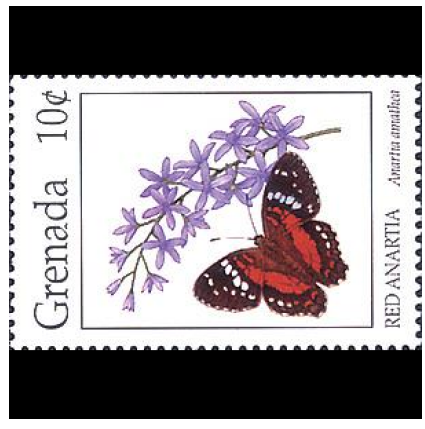

c) $180^{\circ}$

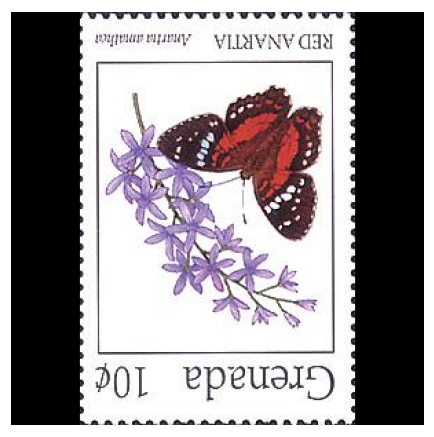

d) $270^{\circ}$

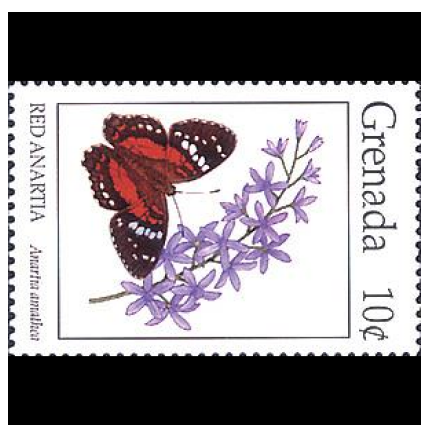

Fig. 3. Original image and its rotated versions used in the numerical experiment

a) Sample image

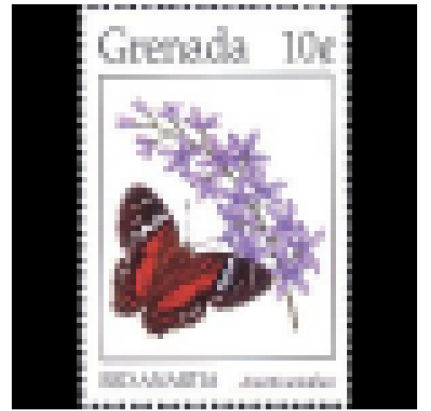

c) $\mathrm{SNR}=-3.5 \mathrm{~dB}$

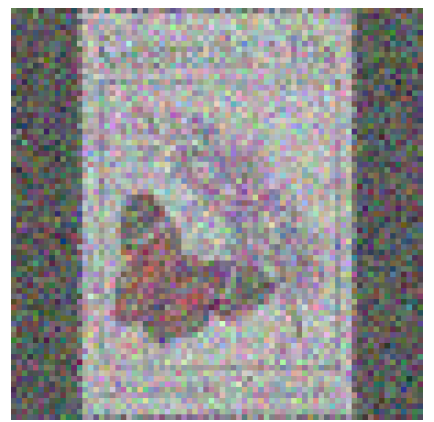

d) $\mathrm{SNR}=-6.5 \mathrm{~dB}$

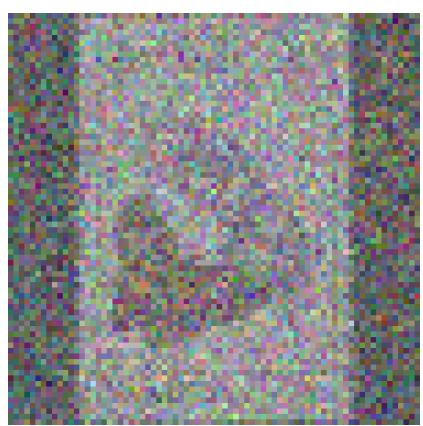

Fig. 4. A compressed image and its noisy versions 
Table 1

Noise standard deviations used in simulation

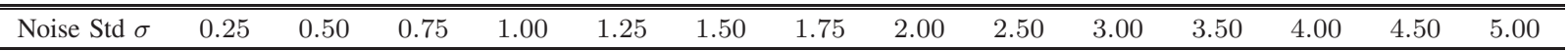

We chose the noise standard deviations given in Table 1 for our simulation. For each noise standard deviation, 12, 000 trials were conducted and the number of errors recorded. The results are presented in Figs. 5, 6, and 7. In Fig. 5, the error rate, which is the number of errors divided by the number of trials, of the simulation using the original prototype images is plotted against the SNR. In Fig. 6, we plot the error rate of the simulation using the compressed images. A comparison of the error rates for the original and compressed images is given in Fig. 7. In the plots, the SNR is defined using the average signal standard deviation $\sigma=0.45$ of all prototype images.

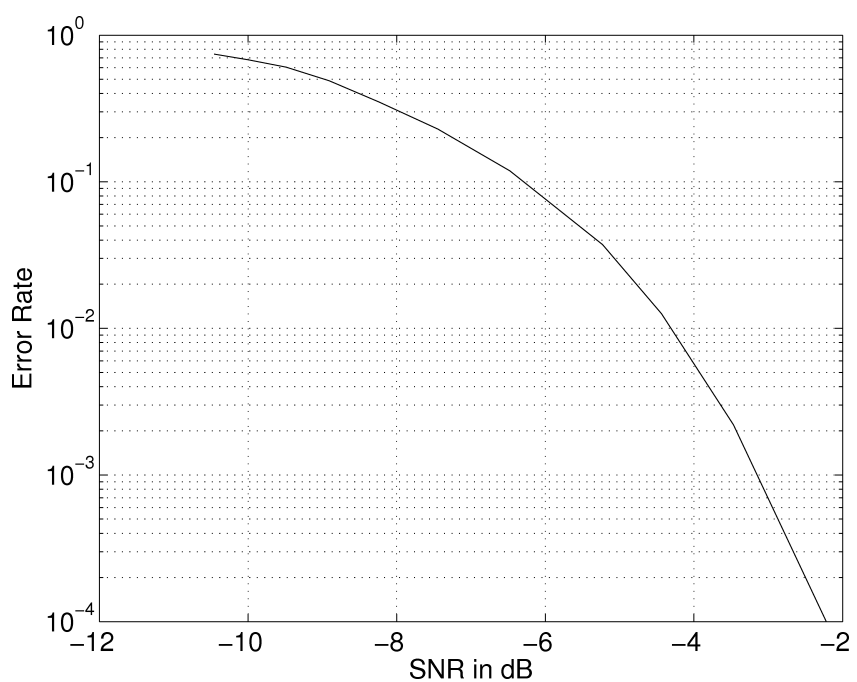

Fig. 5. A plot of the error rate versus signal-to-noise ratio - original images

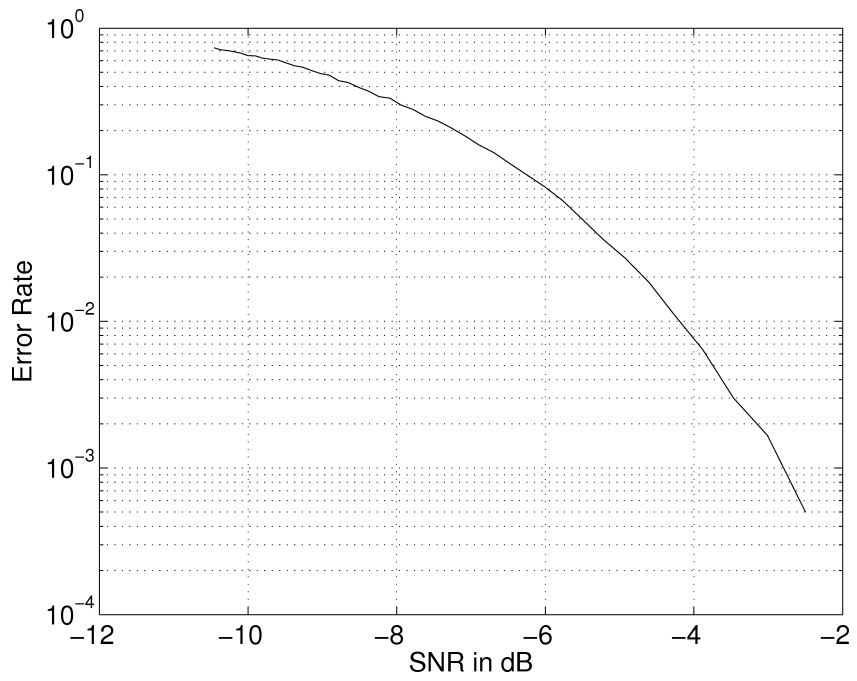

Fig. 6. Error rate versus signal-to-noise ratio - compressed images

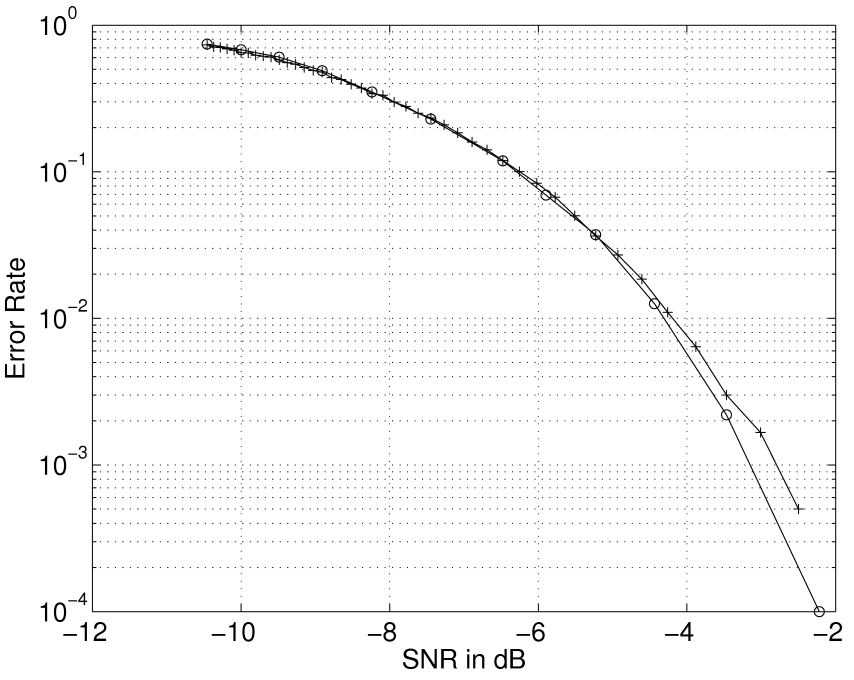

Fig. 7. Error rate versus signal-to-noise ratio - comparison for the compressed images and uncompressed images

Remark 2. We also performed the same experiments with only rotation but no noise. The algorithm was able to identify all presented images correctly and there were no errors. This is exactly as the theory predicted and this also shows that $d_{P}$ is indeed a metric on the set of prototype images.

\section{Fourier descriptor}

In this section, we discuss the difference between our proposed method and the well-known Fourier descriptor technique, which also uses the coefficients of the DFT. The Fourier descriptors are used for boundary curve and shape classifications, see, for example, Zahn and Roskies [17], Persoon and Fu [18], and Krzyżak, Leung, and Suen [19]. To facilitate the discussion, we briefly review the basic Fourier descriptor method.

Let $\Omega$ denote a shape in $\mathbb{R}^{2}$ with a well-defined boundary $\partial \Omega$. In the context of the Fourier descriptor method, $\mathbb{R}^{2}$ is identified with the complex plane $\mathbb{C}$ via

$$
(x, y) \leftrightarrow z=x+j y .
$$

From $\partial \Omega$, a finite number of points $\left\{z_{0}, \ldots, z_{N-1}\right\}$ are chosen to give a good approximation of $\partial \Omega$. The Fourier descriptors of the boundary $\partial \Omega$ are the DFT coefficients of $\left\{z_{0}, \ldots, z_{N-1}\right\}$ :

$$
\widehat{z}_{n}=\sum_{m=0}^{N-1} z_{m} e^{-2 \pi j m \frac{n}{N}}, \quad n=0, \ldots, N-1 .
$$

Note that some authors use the normalization factor $1 / N$ in the definition of the DFT but that clearly makes no difference. The Fourier descriptors have a number of nice properties, including translation invariance, proportional scaling, and rotation and phase relation; the details can be found in the above 
references. Typically, the Fourier descriptors are compared to prototypes for classification. The number of points used can be reduced by omitting the high frequency components of the DFT. This causes the loss of fine details but the overall shape is retained. However, the order of the coefficients themselves must be same.

The most fundamental difference between the Fourier descriptor method and the method proposed in this paper is that the input data $\left\{z_{0}, \ldots, z_{N-1}\right\}$ for the Fourier descriptor method are the coordinates of chosen points on $\partial \Omega$, which means that the Fourier descriptors encode positional information since they are the DFT coefficients of coordinates. On the other hand, we use, in our method, the color and intensity of each pixel of an image and the location of each pixel is implicit in the lattice structure used in the DFT. Since the input data used in the two methods are completely different in nature, the methods are fundamentally different.

Since our proposed method does not use positional information, the question of scaling and rotation must be addressed differently and it is an interesting problem to find an appropriate mathematical structure to answer these questions in a practical manner. One possible approach is to embed the various geometrically transformed images in a large lattice structure.

Also, the order information is important in Fourier descriptors while in our technique, we only use the magnitude information of the DFT without using any order information. Our technique can also be extended to work in higher dimensions. For example, a two dimensional color image can be consider as a three dimensional object.

\section{Conclusions}

We presented a Fourier transform based pattern recognition algorithm. The algorithm uses the magnitudes of a small number of Fourier coefficients of an image as its signature vector. We presented mathematical justifications as to why using a small number of Fourier coefficients as a signature vector may be better than using all Fourier coefficients in the presence of noise. Simulations were conducted to demonstrate the effectiveness of the algorithm in noisy environments.

\section{REFERENCES}

[1] J. Altmann and H.J.P. Reitböck, "A fast correlation method for scale- and translation-invariant pattern recognition", IEEE Trans. on Pattern Analysis and Machine Intelligence PAMI-6 (1), 46-57 (1984).
[2] H.J. Reitboeck and J. Altmann, "A model for size- and rotationinvariant pattern processing in the visual system", Biological Cybernetics 51 (2), 113-121 (1984).

[3] P.H. Gardenier, B.C. McCallum, and R.T. Bates, "Fourier transform magnitudes are unique pattern recognition templates", Biological Cybernetics 54 (6), 385-391 (1986).

[4] G.Y. Chen, T.D. Bui, and A. Krzyżak, "Invariant pattern recognition using radon, dual-tree complex wavelet and Fourier transforms", Pattern Recognition 42 (9), 2013-2019 (2009).

[5] R. Barakat and G. Newsam, "Necessary conditions for a unique solution to two-dimensional phase recovery", J. Mathematical Physics 25 (11), 3190-3193 (1984).

[6] P.L. Van Hove, J.S. Lam, and A.V. Oppenheim, "Signal reconstruction from Fourier transform amplitude", in Applications of Digital Image Processing IV, ed. A.G. Tescher, pp. 214225, SPIE - The International Society for Optical Engineering, Washington, 1982.

[7] L.S. Taylor, "The phase retrieval problem", IEEE Trans. on Antennas and Propagation AP-29 (2), 386-391 (1981).

[8] E.J. Akutowicz, "On the determination of the phase of a Fourier integral, I", Trans. American Mathematical Society 83, 179 192 (1956).

[9] E.J. Akutowicz, "On the determination of the phase of a Fourier integral, II", Proc. American Mathematical Society 8, 234 238 (1957).

[10] M.H. Hayes, J.S. Lim, and A.V. Oppenheim, "Signal reconstruction from phase or magnitude", IEEE Trans. on Acoustics, Speech, and Signal Processing ASSP-28 (6), 672-680 (1980).

[11] M.H. Hayes, "The reconstruction of a multidimensional sequence from the phase or magnitude of its Fourier transform", IEEE Trans. on Acoustics, Speech, and Signal Processing ASSP-30 (2), 140-154 (1982).

[12] R.H.T. Bates and M.J. McDonnell, Image Restoration and Reconstruction, Oxford University Press, New York, 1986.

[13] H. Dym and H.P. McKean, Fourier Series and Integrals, Academic Press, New York, 1972.

[14] J.S. Walker, Fourier Analysis, Oxford University Press, New York, 1988.

[15] R.C. Gonzalez and P. Wintz, Digital Image Processing, Addison-Wesley Publishing Company, Inc., Reading, Massachusetts, 1977.

[16] C. Oh and S.H. Żak, "Image recall using a large scale generalized Brain-State-in-a-Box neural network", Int. J. Applied Mathematics and Computer Science 15 (1), 99-114 (2005).

[17] C.T. Zahn and R.Z. Roskies, "Fourier descriptors for plane closed curves", IEEE Trans. on Computers C-21, 269-281 (1972).

[18] E. Persoon and K.S. Fu, "Shape discrimination using Fourier descriptors", IEEE Trans. On Systems, Man and Cybernetics 7 (3), 170-179 (1977).

[19] A. Krzyżak, S.Y. Leung, and C.Y. Suen, "Reconstruction of two-dimensional patterns from Fourier descriptors", Machine Vision and Applications 2 (3), 123-140 (1989). 